\title{
Diagnosis of Difficult Breast Lesions by Multimode Ultrasound: A Case Report
}

\author{
Feiqian Wang and Litao Ruan* \\ Director of Ultrasound department, The First Affiliated Hospital of Xi'an Jiaotong University, China
}

*Corresponding author: Litao Ruan, Director of Ultrasound department, The First Affiliated Hospital of Xi'an Jiaotong University, 277 Yanta West Road, Xi'an, Shaanxi 710061, P.R. China

\begin{tabular}{|c|c|}
\hline ARTICLE INFO & ABSTRACT \\
\hline Received: 㟉 July 08, 2019 & Citation: Feiqian Wang, Litao Ruan. Diagnosis of Difficult Breast Lesions by Multimode \\
\hline Published: 幽 July 15, 2019 & Ultrasound: A Case Report. Biomed J Sci \& Tech Res 19(4)-2019. BJSTR. MS.ID.003330. \\
\hline
\end{tabular}

\section{Case Report}

A 42-year-old woman was found to have a lesion in left breast by follow up conventional ultrasound 1 year after left breast operation of invasive carcinoma (about 14 and $7 \mathrm{~mm}$ in size). The surgical method of breast cancer was local resection, the resection range was $5 \times 4 \times 4 \mathrm{~cm}$, unfilled. Postoperative radiotherapy was performed with DT50Gy for 25 times. Half a year after operation, ultrasound scan discovered an extremely low echo area at 2 o'clock below the left breast incision with the size of about 7 and $6 \mathrm{~mm}$. According to the characteristic of the clear boundary and equal internal area in ultrasound imaging, this area was diagnosed as wrapped fluid originated from breast operation. In this visit, in contrast, it was found that there was a heterogeneous hypoechoic lesion at the 2 o'clock incision of the left breast, the size was about 9 and $7 \mathrm{~mm}$. The boundary was not clear while the shape was irregular. Particularly, there is coarse calcification in and shadow behind the lesion. Color Doppler US images showed no blood flow signal in or surrounded the lesion. There was no obvious abnormality detected in double axillary lymph nodes. At the time of this visit, the specialist examination showed that the left breast incision was slightly above the surface of skin. Mild pain was complained during local compression at the lesion. No nipple discharge was found. No abnormally enlarged lymph nodes in armpit was touched. The patient underwent compression elastography, which showed that the texture of the hypoechoic node was hard, the internal hardness was uneven, and the texture of a small part of the tissue around the lesion was also hard. The elastic imaging score was assessed as grade 4. BI-RADS 4c was classified in terms of the imaging of ultrasound and elastography. Considering the recurrence of breast cancer, puncture biopsy was recommended by ultrasound doctor. Contrast-enhanced ultrasonography (CEUS) was performed to further confirm the diagnosis. Contrast agent of $5.0 \mathrm{ml}$ was shot injected into the median cubital vein. There was no contrast medium found filling in the lesion. Unfortunately, the whole breast has a low perfusion in CEUS which make the observation between the lesion and surroundings uneasy. To make the results more definitely, the micro flow exhibit mode (MFE) of Chinese Miley Resona 7 ultrasound diagnostic instrument was used. With that mode, no contrast medium was found in the lesion. The boundary of the lesion was clear, and the range did not change when doing CEUS. In order to avoid the effect of a small amount of displacement caused by respiration and chest wall movement on contrast observation, we used Mixed mode of Gray scale ultrasound and CEUS for further observation. With that mixed mode, absolutely no contrast agent was found in the lesion. According to the results of CEUS, the lesion was classified as BI-RADS 4a grade. The lesion was considered to be cystic in view of the previous history of encapsulated fluid in the surgical area. Ultrasound-guided biopsies were performed, and the pathological result was focal fat necrosis wrapped in breast fibers.

Fat liquefaction and necrosis is a non-suppurative inflammatory reaction of adipose tissue. It could be due to over-stimulation and amputation of the main blood supply artery in operation (such as incision traction, electric knife thermal burn, excessive incision traction, ligation and clamping). Local radiotherapy after operation is also an inducement. All these malpractices may lead to intraoperative and postoperative adipose tissue destruction, decomposition, oxidation or aseptic inflammation. Ultrasound findings of breast fat liquefaction are diverse. It can be ranged from anechoic with well-defined circumscription, smooth margin and internal homogeneous content, hypoechoic with clear boundary and uniform or heterogeneous internal echo, mixed echo with 
clear boundary to mixed echo with vague boundary and posterior shadow. When the images are the last four cases, it is difficult to distinguished from benign and malignant lesion. Information such as the history of breast cancer, lesions below the incision, ultrasound appearance of unclear boundary, posterior shadow and calcification can easily mislead doctors into being misdiagnosed as breast cancer recurrence. It is worth noting that CEUS has important value in differential diagnosis of this kind of lesions. Importantly, considering the individual differences of contrast-enhanced ultrasound, contrast-enhanced ultrasound may sometimes not be effective. In that state, attention should be paid to the application of new functions of ultrasonic instruments to improve the effect of contrast. CEUS appearance of "completely no enhancement" and "clear boundary" is an important discrimination point. In order to make a correct and accurate diagnosis, it is necessary to carefully analyze the history of patients receiving radiation therapy and the history of early postoperative fluid entrapment (Figures 1-5).

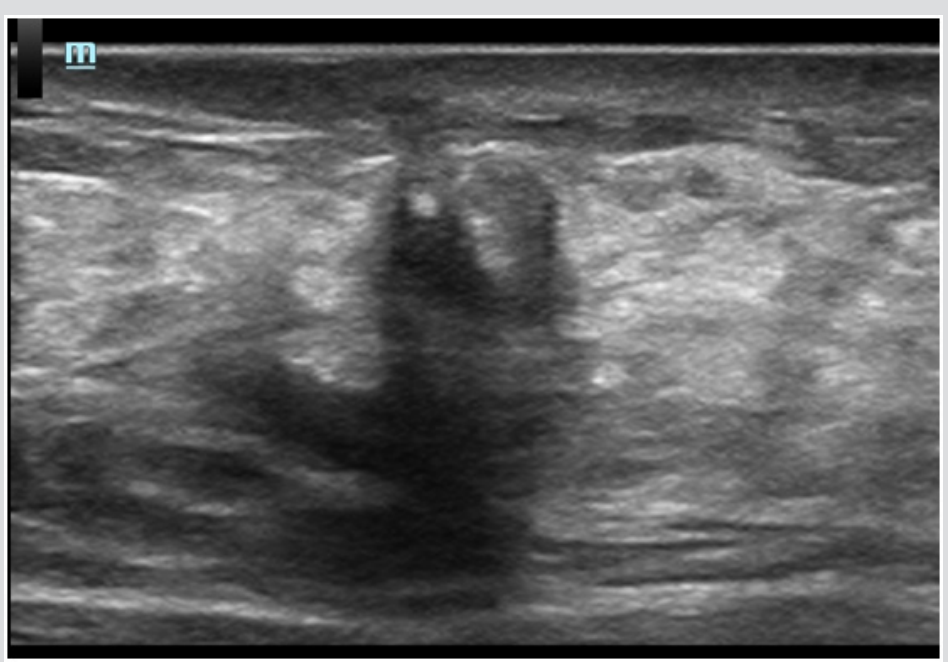

Figure 1: Gray scale ultrasound imaging of the lesion.

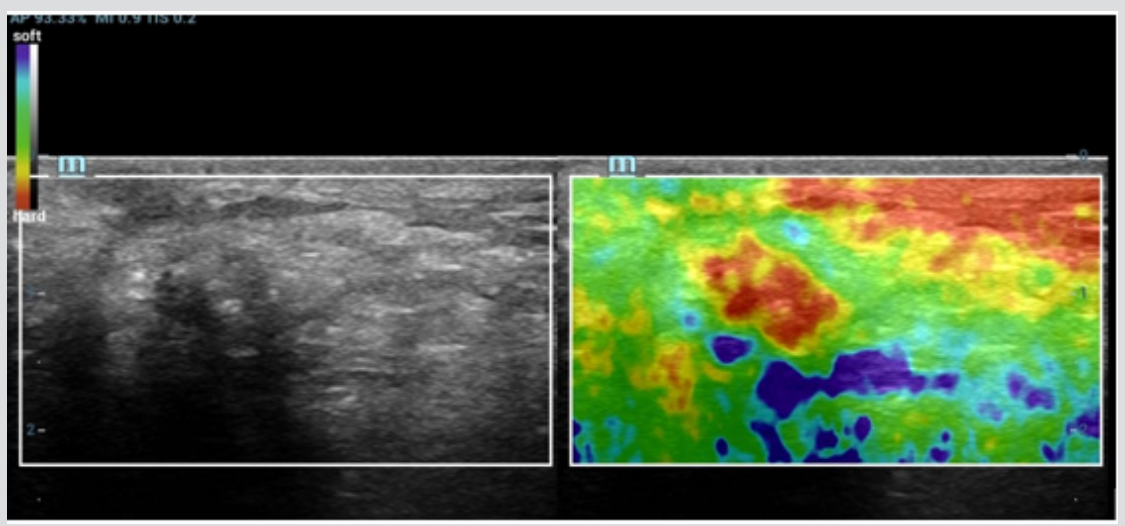

Figure 2: Ulastography imaging of the lesion.

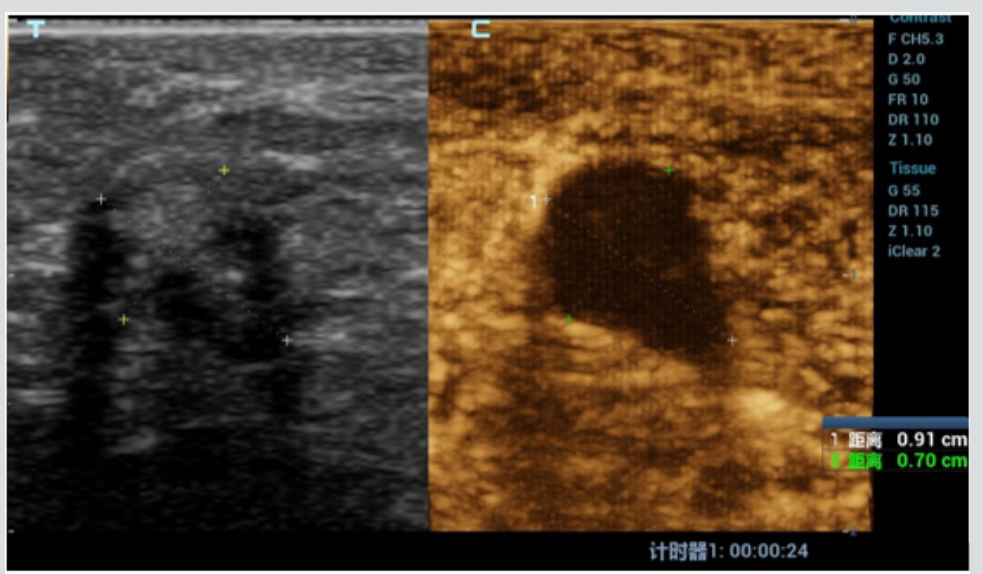

Figure 3: Micro flow exhibit (MFE) mode in CEUS. 


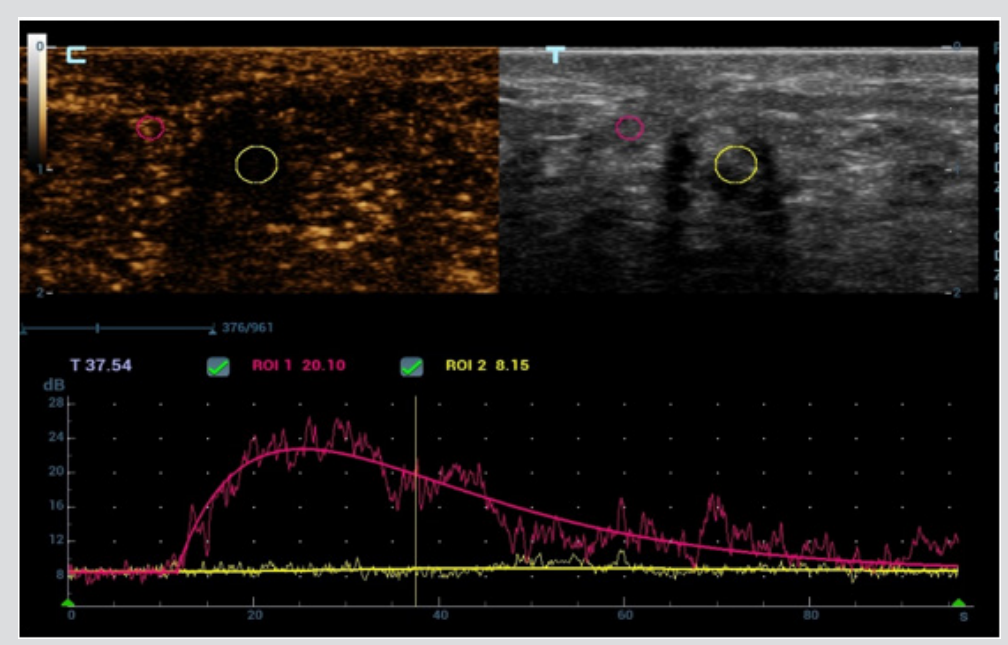

Figure 4: Time intensity curve of CEUS.

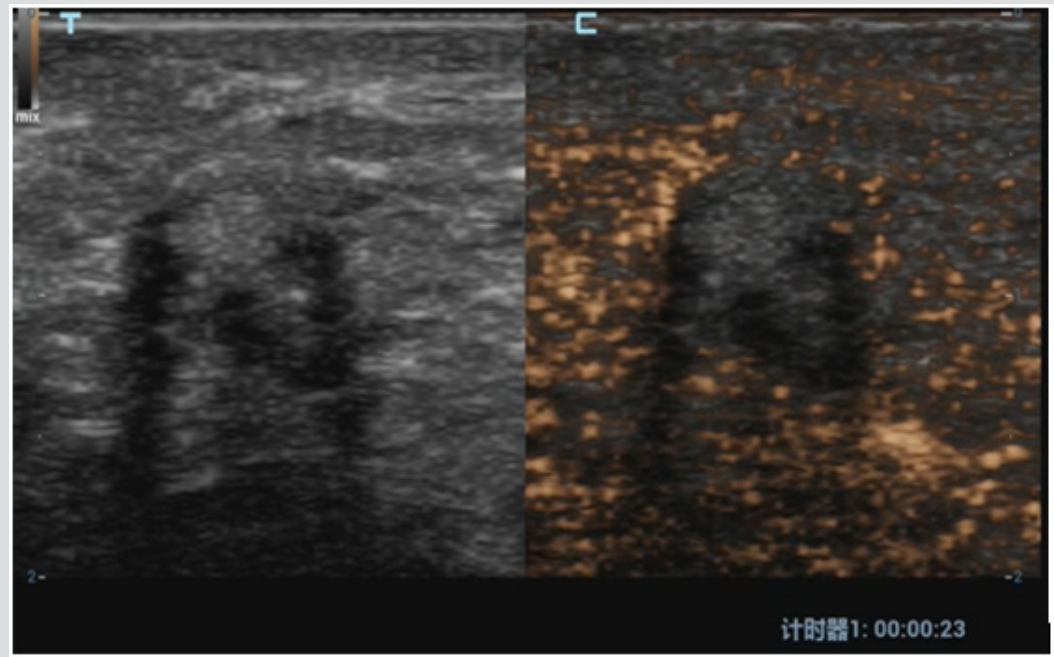

Figure 5: Mixed mode of Gray scale ultrasound and CEUS.

\section{Acknowledgement}

None.

\section{ISSN: 2574-1241}

DOI: 10.26717/BJSTR.2019.19.003330

Litao Ruan. Biomed J Sci \& Tech Res

(c) (P) This work is licensed under Creative

Submission Link: https://biomedres.us/submit-manuscript.php

\section{Conflict of Interest}

No conflict of interest.

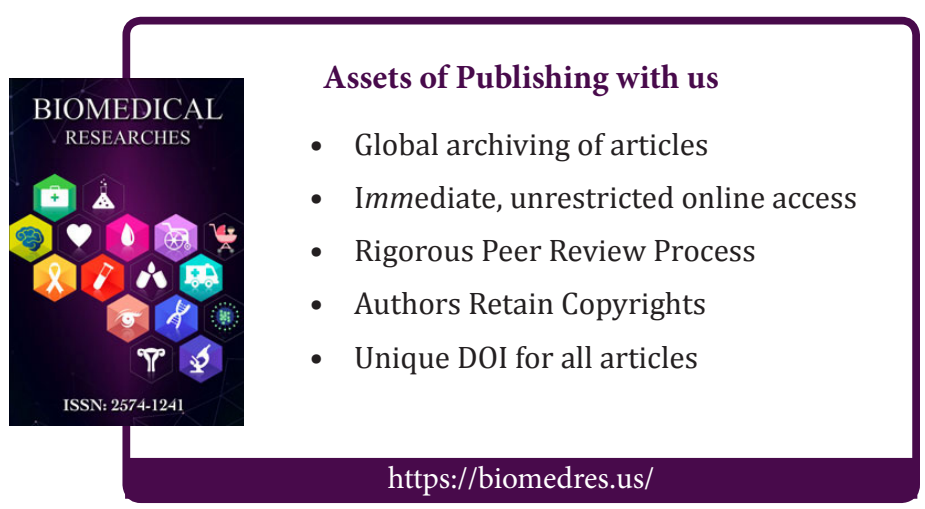

